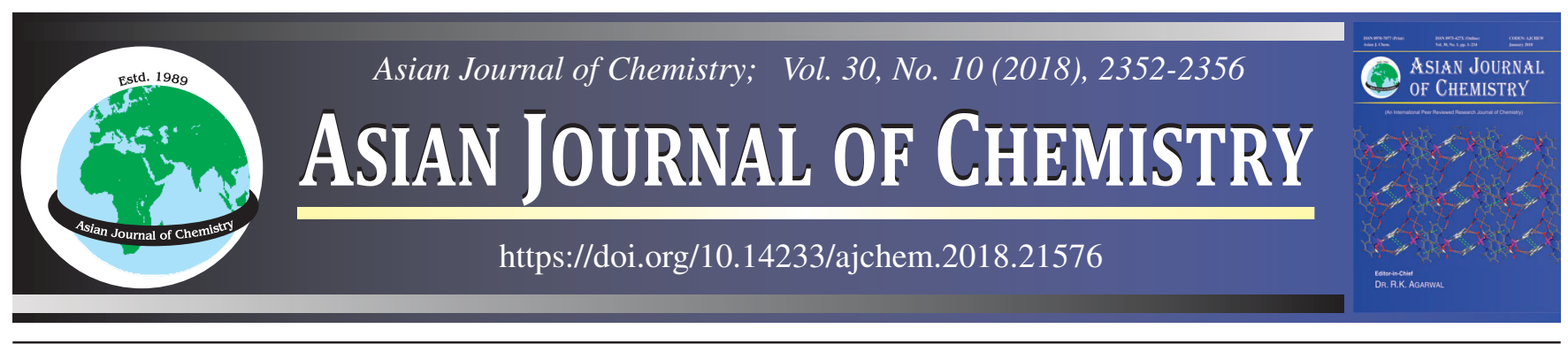

\title{
A Rapid Phase Transfer Catalyzed Heteroarylation by C-O Coupling and Ring Opening of Isopropylidene Glycerol in Synthesis of (Pyrazolopyrimidin-4-yloxy)propane-1,2-diols
}

\author{
Vivek C. Ramani, NiRmal M. Shah and Rina D. Shah*
}

Department of Chemistry, M.G. Science Institute, Ahmedabad-380 006, India

*Corresponding author: E-mail: drrdshah@yahoo.co.in

Received: 12 July 2018;

Accepted: 21 August 2018;

Published online: 31 August 2018;

AJC-19073

A comparative study of C-O cross coupling reaction in synthesis of 4-(O,O-isopropylidene-2,3-dihydroxypropoxypyrazolo[3,4- $d$ ]pyrimidines (3) has been under taken using phase transfer catalysis. Liquid-liquid phase transfer catalysis has been explored in reaction between (2,2-dimethyl-1,3-dioxolan-4-yl)methanol (1) and 4-chloropyrazolo[3,4- $d$ ]pyrimidines (2) using tetrabutylammonium bromide (TBAB) as phase transfer catalyst, aqueous $\mathrm{NaOH}$ as inorganic phase and chlorobenzene as organic phase at room temperature to form 3 . Ring opening of (2,2-dimethyl-1,3-dioxolan-4-yl)methanolyl present in $\mathbf{3}$ under acidic hydrolysis at room temperature afforded 3-(1,6disubstituted $1 H$-pyrazolo[3,4- $d]$ pyrimidin-4-yloxy)propane-1,2-diols (4).

Keywords: C-O cross coupling, O-arylation, Liquid-liquid phase transfer catalysis, Isopropylidene glycerol.

ᄂ - - - - - - - - - - - - - - - - - - - - - - - - - - - -

\section{INTRODUCTION}

Phase transfer was used to catalyze nucleophilic aromatic substitution by Makosza and other [1-3]. Zoltewicz and others [4-7] have given a good early review of various methods and compared with other techniques that make use of polar solvents, transition metals and monoelectron transfers. In general, phase transfer catalysis (PTC) can be used for heteroaromatic SN reactions [8-11]. Chloro group present at position 2 and 4 in pyrimidine has been found reactive towards SN reactions with variety of nucleophilic reagents $[12,13]$. Nucleophilic displacements under phase transfer catalysis in fused pyrimidines have also been reported [14-16]. C-O coupling producing ether linkage (O-alkylation, $\mathrm{O}$-arylation) is an important example of $\mathrm{SN}$ reaction. Isopropylidine glycerol moiety was known to impart remarkable biological activities [17-29]. In addition pyrimidine and fused pyrimidines with glyceryl moiety showing the pharmaceutical significance have been reported [30,31]. Synthesis of pyrazolopyrimidine having isopropylidene moiety are less reported [31]. Incorporation of phase transfer catalysis with Williamson ether synthesis provided a smoother and convenient path for ether formation. Few cases for heteroaromatic SN reactions employing alkoxide ions under phase transfer catalysis in heteroaryl halides have been reported. Pyrazolopyrimidines have received special attention because pyrazolopyrimidines found to be the structural analogous of biogenic purine class compounds, having high impact in the field of pharmaceuticals with vast spectrum of biological activities. Moreover, to resolve environmental issues the present need is to replace classical methods by environmental friendly techniques.

\section{EXPERIMENTAL}

All the reagents used were purchased from S.D. fine chemicals and Aldrich Chemicals (LR grade). Melting points were uncorrected and determined on Toshniwal melting point apparatus in open capillary tubes. IR $(\mathrm{KBr}) \operatorname{spectra}\left(\mathrm{v}_{\max }, \mathrm{cm}^{-1}\right)$ of compounds 3 and $\mathbf{4}$ were recorded on Buck-500 spectrophotometer in the form of $\mathrm{KBr}$ pellets and. ${ }^{1} \mathrm{H} \mathrm{NMR}$ and ${ }^{13} \mathrm{C}$ NMR were recorded on Bruker spectrometer at $400 \mathrm{MHz}$ in DMSO/DMSO- $d_{6}$ using TMS as reference and the values are given in $\delta \mathrm{ppm}$. The mass spectra were recorded on LC-MSAgilent 1100 series. The purity of compounds $\mathbf{3}$ and $\mathbf{4}$ was checked routinely by TLC using $n$-hexane:ethyl acetate $(8: 2)$ using silica gel-G plates and spots were visualized by exposing the dry plates in iodine vapours and UV light $(\lambda=254 \mathrm{~nm})$.

In present report, $\mathrm{C}-\mathrm{O}$ coupling between pharmaceutically accepted (2,2-dimethyl-1,3-dioxolan-4-yl)methanol (1) and

This is an open access journal, and articles are distributed under the terms of the Creative Commons Attribution-NonCommercial 4.0 International (CC BY-NC 4.0) License, which allows others to copy and redistribute the material in any medium or format, remix, transform, and build upon the material, as long as appropriate credit is given and the new creations are licensed under the identical terms. 


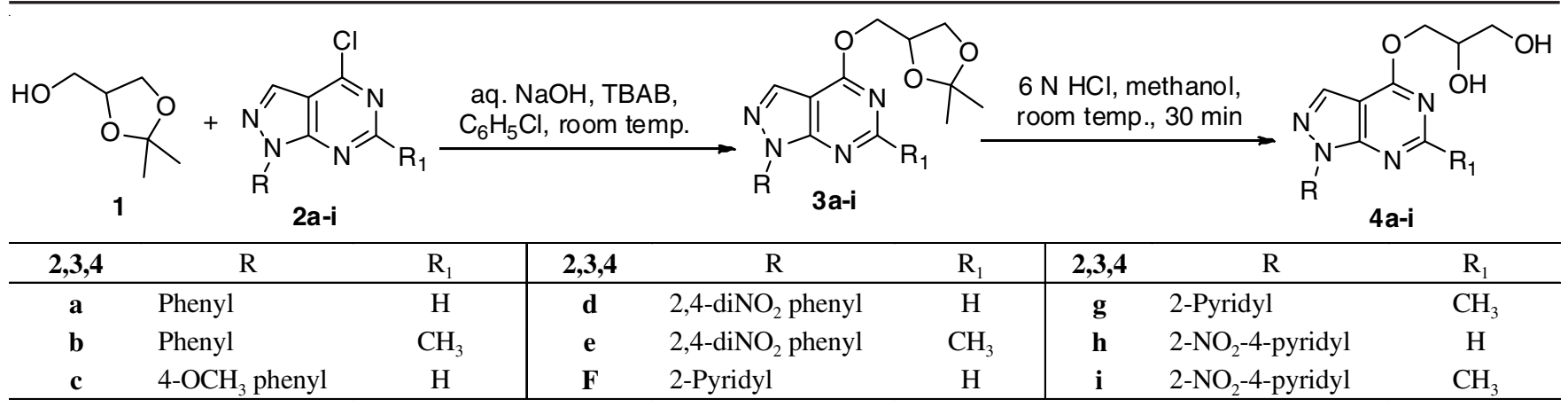

Scheme-I

1,6-disubstituted 4-chloropyrazolo[3,4- $d$ ]pyrimidines (2) has been attempted under liquid-liquid phase transfer catalysis at room temperature using a mixture of chlorobenzene and sodium hydroxide solution $(50 \% \mathrm{w} / \mathrm{v})$ as solvent and tetrabutylammonium bromide (TBAB) as a phase transfer catalyst to form 4(O,O-isopropylidene-2,3-dihydroxypropoxy)pyrazolo[3,4$d$ ]pyrimidines (3). Compound $\mathbf{3}$ when subjected to acid hydrolysis with alcoholic $\mathrm{HCl}$ at room temperature under stirring underwent ring opening to give 3-(1,6-substituted $1 H$-pyrazolo[3,4-d]pyrimidin-4-yloxy)propane-1,2-diols (4) in excellent yield (Scheme-I).

General method for synthesis of 4-((2,2-dimethyl-1,3dioxolan-4-yl)methoxy)-1,6-disubstituted $1 H$-pyrazolo[3,4d]pyrimidines (3a-h): To a solution of sodium hydroxide (50\% w/v, $3 \mathrm{~mL}$ ), TBAB (5 mmol, $0.161 \mathrm{~g})$, chlorobenzene (15 mL) and 1,6-disubstituted 4-chloropyrazolo[3,4- $d$ ]pyrimidines [30,32,33] $(\mathbf{2}, 0.005 \mathrm{~mol}, 1.45 \mathrm{~g})$ was added $d l$-(2,2-dimethyl1,3-dioxolan-4-yl)methanol (2,0.006 mol, $0.792 \mathrm{~g})$. The mixture was stirred at room temperature and the completion of reaction (25-35 min) was determined by TLC (benzene:ethanol, 8:2). The water $(25 \mathrm{~mL})$ was added to the reaction mixture and stirring was continued for $5 \mathrm{~min}$. The phases were then separated and the aqueous phase was extracted with chlorobenzene $(15 \mathrm{~mL})$. The combined organic layers were dried over anhydrous magnesium sulphate and chlorobenzene was removed in vacuo to obtain the crude solid product which was crystallized from ethanol.

1-Phenyl-4-((2,2-dimethyl-1,3-dioxolan-4-yl)methoxy)$\mathbf{1 H}$-pyrazolo[3,4- $\boldsymbol{d}]$ pyrimidine (3a): Reaction time: $25 \mathrm{~min}$, yield: $90 \%$, m.p.: $232-234{ }^{\circ} \mathrm{C}$, IR $\left(\mathrm{KBr}, v_{\max }, \mathrm{cm}^{-1}\right): 3010$, 2980, $2870(\mathrm{CH}), 1588,1545(\mathrm{C}=\mathrm{C}, \mathrm{C}=\mathrm{N}),{ }^{1} \mathrm{H}$ NMR ((DMSO$\left.d_{6}\right): \delta 1.31-1.32\left(\mathrm{~m}, 6 \mathrm{H}, \mathrm{CH}_{3}\right), 4.03-4.08\left(\mathrm{~m}, 2 \mathrm{H}, \mathrm{CH}_{2}\right), 4.27-$ $4.33\left(\mathrm{~m}, 2 \mathrm{H}, \mathrm{CH}_{2}\right), 4.54-4.61(\mathrm{~m}, 1 \mathrm{H}, \mathrm{CH}), 8.38$ (s, 2H, Ar$\mathrm{H}), 8.60-8.62$ (d, $J=6.0 \mathrm{~Hz}, 2 \mathrm{H}, \mathrm{Ar}-\mathrm{H}), 8.77-8.78$ (d, $J=6.0$ $\mathrm{Hz}, 2 \mathrm{H}, \mathrm{Ar}-\mathrm{H}$ ), 9.28 (s, H, Ar-H at $\mathrm{C}_{3}$ ), ${ }^{13} \mathrm{C}$ NMR (DMSO- $d_{6}$ ): $\delta$ 163.22, 152.38, 148.71, 146.78, 143.21, 132.73, 130.32, 115.65, 112.50, 101.17, 63.12, 60.16, 14.29 MS: $\mathrm{M}^{+}(326)$ : Analysis (\%) for $\mathrm{C}_{17} \mathrm{H}_{18} \mathrm{~N}_{4} \mathrm{O}_{3}$ Calcd. C, 62.57; H, 5.56; N, 17.17 \%. Found: C, 62.37; H, 5.27; N, $17.56 \%$.

1-Phenyl-4-((2,2-dimethyl-1,3-dioxolan-4-yl)methoxy)6-methyl-1H-pyrazolo[3,4- $\boldsymbol{d}]$ pyrimidine (3b): Reaction time: 25 min, yield: $92 \%$, m.p.: 221-223 ${ }^{\circ} \mathrm{C}$, IR $\left(\mathrm{KBr}, v_{\max }, \mathrm{cm}^{-1}\right)$ : 3008, 2982, $2871(\mathrm{CH}), 1590,1580(\mathrm{C}=\mathrm{C}, \mathrm{C}=\mathrm{N}),{ }^{1} \mathrm{H}$ NMR ((DMSO-d $)_{6}: \delta 1.31-1.32\left(\mathrm{~m}, 6 \mathrm{H}, \mathrm{CH}_{3}\right), 2.62\left(\mathrm{~s}, 3 \mathrm{H}, \mathrm{Ar}-\mathrm{CH}_{3}\right)$, 4.05-4.08 (m, 2H, $\left.\mathrm{CH}_{2}\right), 4.28-4.33\left(\mathrm{~m}, 2 \mathrm{H}, \mathrm{CH}_{2}\right), 4.54-4.61$ (m, 1H, CH), 8.38 (s, 2H, Ar-H), 8.60-8.62 (d, J = 6.0 Hz, 2H, Ar-H), 8.77-8.79 (d, $J=6.0 \mathrm{~Hz}, 1 \mathrm{H}, \mathrm{Ar}-\mathrm{H}$ at $\mathrm{C}_{6}$ ), 9.07 (s, 1H, $\mathrm{Ar}-\mathrm{H}),{ }^{13} \mathrm{C}$ NMR (DMSO- $d_{6}$ ): 164.74, 153.71, 148.67, 146.17, 143.12, 132.77, 131.13, 114.15, 102.40, 61.18, 21.15.14. MS: $\mathrm{M}^{+}$(340): Analysis (\%) for $\mathrm{C}_{18} \mathrm{H}_{20} \mathrm{~N}_{4} \mathrm{O}_{3}$ Calcd.: C, 63.52; $\mathrm{H}$, $5.92 ;$ N, $16.46 \%$. Found: C, 58.45; H, 5.79; N, $17.33 \%$.

1-(4-Methoxyphenyl)-4-((2,2-dimethyl-1,3-dioxolan-4yl)methoxy)-1H-pyrazolo[3,4- $d]$ pyrimidine (3c): Reaction time: 25 min, yield: $95 \%$, m.p.: $248-250{ }^{\circ} \mathrm{C}$, IR (KBr, $v_{\max }$, $\left.\mathrm{cm}^{-1}\right)$ : 3012, 2978, $2875(\mathrm{CH}), 1590,1580(\mathrm{C}=\mathrm{C}, \mathrm{C}=\mathrm{N}),{ }^{1} \mathrm{H}$ NMR ((DMSO-d $\left.d_{6}\right): \delta$ 1.32-1.34 $\left(\mathrm{m}, 6 \mathrm{H}, \mathrm{CH}_{3}\right), 3.86(\mathrm{~s}, 3 \mathrm{H}$, $\left.\mathrm{OCH}_{3}\right), 4.03-4.08$ (m, 2H, $\left.\mathrm{CH}_{2}\right), 4.32-4.37$ (m, 2H, $\left.\mathrm{CH}_{2}\right)$, 4.544.61 (m, 1H, CH), 8.39 (s, 1H, Ar-H), 8.61-8.62 (d, $J=6.0$ $\mathrm{Hz}, 1 \mathrm{H}, \mathrm{Ar}-\mathrm{H})$ 8.77-8.79 (d, $J=5.6 \mathrm{~Hz}, 2 \mathrm{H}, \mathrm{Ar}-\mathrm{H}), 8.87$ (s, $1 \mathrm{H}, \mathrm{Ar}-\mathrm{H}$ at $\mathrm{C}_{6}$ ), 9.34 (s, $1 \mathrm{H}, \mathrm{Ar}-\mathrm{H}$ at $\mathrm{C}_{3}$ ), ${ }^{13} \mathrm{C}$ NMR (DMSO$\left.d_{6}\right)$ : 163.22, 152.38, 148.75, 146.18, 143.81, 133.73, 130.39, 115.65, 113.51, 101.09, 63.42, 61.11, 55.11, 21.42. MS: $\mathrm{M}^{+}$ (356): Analysis (\%) for $\mathrm{C}_{18} \mathrm{H}_{20} \mathrm{~N}_{4} \mathrm{O}_{4}$ Calcd.: C, 60.66; H, 5.66; N, 15.72. Found: C, 60.85; H, 5.89; N, $17.09 \%$.

1-(2,4-Dinitrophenyl)-4-((2,2-dimethyl-1,3-dioxolan-4yl)methoxy)-1H-pyrazolo[3,4- $d]$ pyrimidine (3d): Reaction time: 30 min, yield: $91 \%$, m.p.: 210-212 ${ }^{\circ} \mathrm{C}$, IR (KBr, $v_{\max }$, $\left.\mathrm{cm}^{-1}\right)$ : 3013, 2988, $2870(\mathrm{CH}), 1578,1555(\mathrm{C}=\mathrm{C}, \mathrm{C}=\mathrm{N}),{ }^{1} \mathrm{H}$ NMR ((DMSO- $\left.d_{6}\right): \delta 1.31-1.32\left(\mathrm{~s}, 6 \mathrm{H}, \mathrm{CH}_{3}\right), 4.03-4.08(\mathrm{~m}$, $\left.2 \mathrm{H}, \mathrm{CH}_{2}\right), 4.32-4.37\left(\mathrm{~m}, 2 \mathrm{H}, \mathrm{CH}_{2}\right), 4.54-4.61(\mathrm{~m}, 1 \mathrm{H}, \mathrm{CH})$, 8.38-8.39 (s, 1H, Ar-H), 8.77-8.79 (d, J = 6.4 Hz, 2H, Ar-H), 9.27 (s, 2H, Ar-H), ${ }^{13} \mathrm{C}$ NMR (DMSO- $d_{6}$ ): 164.24, 153.18, 148.75, 146.78, 143.21, 132.71, 130.93, 113.52, 102.01, 60.98, 13.89. MS: $\mathrm{M}^{+}(416)$ : Analysis (\%) for $\mathrm{C}_{17} \mathrm{H}_{16} \mathrm{~N}_{6} \mathrm{O}_{7}$ Calcd.: $\mathrm{C}$, 49.04; H, 3.87; N, 20.19\%. Found: C, 49.38; H, 4.02; N, $20.58 \%$.

1-(2,4-Dinitrophenyl)-4-((2,2-dimethyl-1,3-dioxolan-4yl)methoxy)-6-methyl-1H-pyrazolo $[3,4-d]$ pyrimidine (3e): Reaction time: 25 min, yield: $95 \%$, m.p.: 204-206 ${ }^{\circ} \mathrm{C}$, IR ( $\mathrm{KBr}$, $\left.v_{\max }, \mathrm{cm}^{-1}\right): 3010,2981,2875(\mathrm{CH}), 1599,1589(\mathrm{C}=\mathrm{C}, \mathrm{C}=\mathrm{N})$, ${ }^{1} \mathrm{H}$ NMR ((DMSO- $\left.d_{6}\right): \delta 1.31-1.32\left(\mathrm{~m}, 6 \mathrm{H}, \mathrm{CH}_{3}\right), 2.63(\mathrm{~s}, 3 \mathrm{H}$, Ar- $\left.\mathrm{CH}_{3}\right), 4.03-4.08$ (m, 2H, CH 2$), 4.32-4.37$ (s, 2H, $\left.\mathrm{CH}_{2}\right)$, 4.544.61 (m, 1H, CH), 8.38-8.40 (d, $J=6.8 \mathrm{~Hz}, 1 \mathrm{H}, \mathrm{Ar}-\mathrm{H})$ 8.77$8.79(\mathrm{~d}, J=4.0 \mathrm{~Hz}, 2 \mathrm{H}, \mathrm{Ar}-\mathrm{H}), 9.16$ (s, 1H, Ar-H at $\left.\mathrm{C}_{3}\right),{ }^{13} \mathrm{C}$ NMR (DMSO-d $_{6}$ : 164.14, 153.14, 148.12, 146.65, 143.95, 132.41, 131.13, 114.78, 102.12, 61.62, 21.45, 14.75. MS: $\mathrm{M}^{+}$ (430): Analysis (\%) for $\mathrm{C}_{18} \mathrm{H}_{18} \mathrm{~N}_{6} \mathrm{O}_{7}$ Calcd.: C, 50.23; $\mathrm{H}, 4.22$; N, $19.53 \%$. Found: C, 50.26; H, 4.36; N, $20.01 \%$.

1-(Pyridin-2-yl)-4-((2,2-dimethyl-1,3-dioxolan-4-yl)methoxy)-1H-pyrazolo[3,4-d]pyrimidine (3f): Reaction time: 30 min, yield: $91 \%$, m.p.: $248-249^{\circ} \mathrm{C}$, IR (KBr, $v_{\max }$, 
$\left.\mathrm{cm}^{-1}\right): 3015,2983,2879(\mathrm{CH}), 1598,1584(\mathrm{C}=\mathrm{C}, \mathrm{C}=\mathrm{N}),{ }^{1} \mathrm{H}$ NMR ((DMSO- $\left.d_{6}\right): \delta 1.31-1.32\left(\mathrm{~m}, 6 \mathrm{H}, \mathrm{CH}_{3}\right), 4.03-4.08(\mathrm{~m}$, $\left.2 \mathrm{H}, \mathrm{CH}_{2}\right), 4.32-4.37\left(\mathrm{~m}, 2 \mathrm{H}, \mathrm{CH}_{2}\right), 4.54-4.60(\mathrm{~m}, 1 \mathrm{H}, \mathrm{CH})$, 8.38 (s, $1 \mathrm{H}, \mathrm{Ar}-\mathrm{H}), 8.61-8.62$ (d, $J=6.0 \mathrm{~Hz}, 1 \mathrm{H}, \mathrm{Ar}-\mathrm{H}), 8.78$ 8.79 (d, $J=6.4 \mathrm{~Hz}, 1 \mathrm{H}, \mathrm{Ar}-\mathrm{H}), 8.99$ (s, 2H, Ar-H) 9.28 (s, 1H, Ar-H at $\mathrm{C}_{3}$ ), ${ }^{13} \mathrm{C}$ NMR (DMSO- $d_{6}$ ): 164.12, 152.48, 149.74, 145.72, 143.71, 133.43, 130.11, 115.65, 112.45, 101.07, 63.78, 60.11, 15.22. MS: $\mathrm{M}^{+}$(327): Analysis (\%) for $\mathrm{C}_{16} \mathrm{H}_{17} \mathrm{~N}_{5} \mathrm{O}_{3}$ Calcd.: C, 58.71; H, 5.23; N, $21.39 \%$. Found: C, 58.95; H, $5.60 ; \mathrm{N}, 21.75 \%$.

1-(Pyridin-2-yl)-4-((2,2-dimethyl-1,3-dioxolan-4yl)methoxy)-6-methyl-1 $H$-pyrazolo $[3,4-d]$ pyrimidine $(3 \mathrm{~g})$ : Reaction time: $35 \mathrm{~min}$, yield: $91 \%$, m.p.: $238-240{ }^{\circ} \mathrm{C}$, IR (KBr, $\left.v_{\max }, \mathrm{cm}^{-1}\right): 3019,2987,2876(\mathrm{CH}), 1586,1566(\mathrm{C}=\mathrm{C}, \mathrm{C}=\mathrm{N})$, ${ }^{1} \mathrm{H}$ NMR ((DMSO- $\left.d_{6}\right): \delta 1.31\left(\mathrm{~m}, 6 \mathrm{H}, \mathrm{CH}_{3}\right), 2.62(\mathrm{~s}, 3 \mathrm{H}, \mathrm{Ar}-$ $\left.\mathrm{CH}_{3}\right)$, 4.03-4.08 (m, $\left.2 \mathrm{H}, \mathrm{CH}_{2}\right) 4.32-4.37\left(\mathrm{~m}, 2 \mathrm{H}, \mathrm{CH}_{2}\right)$, 4.54$4.61(\mathrm{~m}, 1 \mathrm{H}, \mathrm{CH}), 8.38-8.39(\mathrm{~d}, J=6.0 \mathrm{~Hz}, 1 \mathrm{H}, \mathrm{Ar}-\mathrm{H}), 8.60-$ $8.62(\mathrm{~d}, J=6.0 \mathrm{~Hz}, 1 \mathrm{H}, \mathrm{Ar}-\mathrm{H}), 8.77-8.79(\mathrm{~d}, J=6.0 \mathrm{~Hz}, 2 \mathrm{H}$, Ar-H), 9.0 (s, $1 \mathrm{H}, \mathrm{Ar}-\mathrm{H}$ at $\mathrm{C}_{3}$ ), ${ }^{13} \mathrm{C}$ NMR (DMSO- $d_{6}$ ): 163.87 , 152.98, 148.72, 146.95, 144.11, 132.71, 131.13, 114.98, 102.13, 61.99, 21.71, 14.33. MS: $\mathrm{M}^{+}$(341): Analysis (\%) for $\mathrm{C}_{17} \mathrm{H}_{19} \mathrm{~N}_{5} \mathrm{O}_{3}$ Calcd.: $\mathrm{C}, 59.81 ; \mathrm{H}, 5.61 ; \mathrm{N}, 20.52 \%$. Found: C, $59.99 ; \mathrm{H}, 5.81 ; \mathrm{N}, 20.90 \%$.

1-(3-Nitropyridin-4-yl)-4-((2,2-dimethyl-1,3-dioxolan4-yl)methoxy)-1H-pyrazolo[3,4- $d$ ]pyrimidine (3h): Reaction time: 25 min, yield: $90 \%$, m.p.: $256-258{ }^{\circ} \mathrm{C}$, IR $\left(\mathrm{KBr}, v_{\max }\right.$, $\left.\mathrm{cm}^{-1}\right): 3007,2985,2881(\mathrm{CH}), 1590,1556(\mathrm{C}=\mathrm{C}, \mathrm{C}=\mathrm{N}),{ }^{1} \mathrm{H}$ NMR (DMSO- $\left.d_{6}\right): \delta 1.31-1.32\left(\mathrm{~m}, 6 \mathrm{H}, \mathrm{CH}_{3}\right), 4.03-4.08(\mathrm{~m}$, $\left.2 \mathrm{H}, \mathrm{CH}_{2}\right) 4.27-4.37\left(\mathrm{~m}, 2 \mathrm{H}, \mathrm{CH}_{2}\right), 4.54-4.60(\mathrm{~m}, 1 \mathrm{H}, \mathrm{CH})$, 8.38-8.39 (d, $J=6.0 \mathrm{~Hz}, 1 \mathrm{H}, \mathrm{Ar}-\mathrm{H}), 8.77-8.79(\mathrm{~d}, J=6.4 \mathrm{~Hz}$, $2 \mathrm{H}, \mathrm{Ar}-\mathrm{H}$ ), 9.28 (s, $2 \mathrm{H}, \mathrm{Ar}-\mathrm{H}),{ }^{13} \mathrm{C}$ NMR (DMSO- $d_{6}$ ): 164.17, 152.11, 149.98, 145.63, 143.71, 133.49, 130.45, 115.34, 112.62, 101.74, 63.61, 60.41, 15.89. MS: $\mathrm{M}^{+}(372)$ : Analysis (\%) for $\mathrm{C}_{16} \mathrm{H}_{16} \mathrm{~N}_{6} \mathrm{O}_{5}$ Calcd.: C, 51.61; H, 4.33; N, $22.57 \%$. Found: C, 51.89; H, 4.65; N, $23.02 \%$.

1-(3-Nitropyridin-4-yl)-4-((2,2-Dimethyl-1,3-dioxolan4-yl)methoxy)-6-methyl-1H-pyrazolo[3,4- $d]$ pyrimidine (3i): Reaction time: 25 min, yield: $91 \%$, m.p.: $241-243{ }^{\circ} \mathrm{C}$, $\operatorname{IR}\left(\mathrm{KBr}, v_{\max }, \mathrm{cm}^{-1}\right): 3014,2980,2879(\mathrm{CH}), 1595,1563(\mathrm{C}=\mathrm{C}$, $\mathrm{C}=\mathrm{N}),{ }^{1} \mathrm{H}$ NMR ((DMSO- $\left.d_{6}\right): \delta 1.30-1.31\left(\mathrm{~m}, 6 \mathrm{H}, \mathrm{CH}_{3}\right), 2.62$ (s, 3H, Ar- $\mathrm{CH}_{3}$ ), 4.03-4.09 (m, 2H, $\mathrm{CH}_{2}$ ) 4.32-4.36 (m, $2 \mathrm{H}$, $\left.\mathrm{CH}_{2}\right), 4.54-4.60(\mathrm{~m}, 1 \mathrm{H}, \mathrm{CH}), 8.38-8.39(\mathrm{~d}, J=6.0 \mathrm{~Hz}, 1 \mathrm{H}$, Ar-H), 8.77-8.79 (d, $J=6.4$ Hz, 2H, Ar-H) 9.29 (s, 1H, Ar-H at $\mathrm{C}_{3}$ ), ${ }^{13} \mathrm{C}$ NMR (DMSO- $d_{6}$ ): 163.14 152.42, 148.96, 146.12, 144.21, 132.75, 131.12, 114.32, 102.75, 61.98, 21.12, 14.78. MS: $\mathrm{M}^{+}$(386): Analysis (\%) for $\mathrm{C}_{17} \mathrm{H}_{18} \mathrm{~N}_{6} \mathrm{O}_{5}$ Calcd.: C, 52.85; H, 4.70; N, $21.75 \%$. Found: C, 53.12; H, 4.96; N, $22.02 \%$.

General method for synthesis of 3-(1,6-dimethyl-1Hpyrazolo[3,4- $d]$ pyrimidin-4-yloxy)propane-1,2-diols (4a-h): 4-((2,2-Dimethyl-1,3-dioxolan-4-yl)methoxy)-1,6-disubstituted $1 \mathrm{H}$-pyrazolo[3,4- $d$ ] pyrimidines $(\mathbf{3}, 5 \mathrm{mmol})$ were dissolved in methanol $(20 \mathrm{~mL})$ and $6 \mathrm{~N}$ hydrochloric acid $(5 \mathrm{~mL})$ was stirred for $0.5 \mathrm{~h}$ at room temperature. The reaction mixture was poured onto the crushed ice, the solid separated was filtered, washed with water till neutral, dried and crystallized from ethanol to get the titled compound $\mathbf{4}$ as off-white to yellowish-white solid.

3-((1-Phenyl-1 $H$-pyrazolo[3,4- $d$ ]pyrimidin-4-yl)oxy)propane-1,2-diol (4a): Yield: 89 \%, m.p.: $161-162{ }^{\circ} \mathrm{C}$, IR
$\left(\mathrm{KBr}, v_{\max }, \mathrm{cm}^{-1}\right): 3438(\mathrm{OH}), 3030,2944,2852(\mathrm{CH}), 1585$, $1530(\mathrm{C}=\mathrm{C}, \mathrm{C}=\mathrm{N}),{ }^{1} \mathrm{H}$ NMR (DMSO- $\left.d_{6}\right): \delta 3.85(\mathrm{~m}, 3 \mathrm{H}$, $\mathrm{CH}_{2} \mathrm{OH}$ ), 3.97 (s, 2H, CH.OH), 4.31-4.36 (m, 2H, $\left.\mathrm{CH}_{2}\right), 8.18$ (s, 2H, Ar-H), 8.37-8.38 (d, $J=3.2 \mathrm{~Hz}, 2 \mathrm{H}, \mathrm{Ar}-\mathrm{H}), 8.76-8.78$ (d, $J=6.0 \mathrm{~Hz}, 2 \mathrm{H}, \mathrm{Ar}-\mathrm{H}$ at $\left.\mathrm{C}_{6}\right), 9.28\left(\mathrm{~s}, 1 \mathrm{H}, \mathrm{Ar}-\mathrm{H}\right.$ at $\left.\mathrm{C}_{3}\right),{ }^{13} \mathrm{C}$ NMR (DMSO- $d_{6}$ ): 162.28, 153.48, 150.74, 148.29, 143.29, 133.02, 130.83, 116.67, 112.54, 101.07, 63.87, 60.11. MS: $\mathrm{M}^{+}$(286): Analysis (\%) for $\mathrm{C}_{14} \mathrm{H}_{14} \mathrm{~N}_{4} \mathrm{O}_{3}$ Calcd.: C, 58.73; $\mathrm{H}$, 4.93 ; N, $19.57 \%$. Found: C, 59.03; H, 5.19; N, $19.86 \%$.

3-((1-Phenyl-6-Methyl-1H-pyrazolo[3,4-d]pyrimidin4-yl)oxy)propane-1,2-diol (4b): yield: $92 \%$, m.p.: 154-155 ${ }^{\circ} \mathrm{C}, \mathrm{IR}\left(\mathrm{KBr}, v_{\max }, \mathrm{cm}^{-1}\right): 3449(\mathrm{OH}), 3010,2970,2838(\mathrm{CH})$, $1587,1535(\mathrm{C}=\mathrm{C}, \mathrm{C}=\mathrm{N}),{ }^{1} \mathrm{H}$ NMR (DMSO- $\left.d_{6}\right): \delta 2.67(\mathrm{~s}, 3 \mathrm{H}$, $\mathrm{CH}_{3}$ ), 3.71-3.85 (m, 3H, $\left.\mathrm{CH}_{2} \mathrm{OH}\right), 3.98$ (s, $2 \mathrm{H}, \mathrm{CH}_{-2}$ ), 4.324.39 (m, 2H, CHOH), 8.38 (s, 2H, Ar-H), 8.58-8.59 (d, $J=$ $3.6 \mathrm{~Hz}, 2 \mathrm{H}, \mathrm{Ar}-\mathrm{H}), 8.87-8.88$ (d, $J=6.4 \mathrm{~Hz}, 1 \mathrm{H}, \mathrm{Ar}-\mathrm{H}), 9.07$ (s, $1 \mathrm{H}, \mathrm{Ar}-\mathrm{H}$ at $\mathrm{C}_{3}$ ), ${ }^{13} \mathrm{C}$ NMR (DMSO- $d_{6}$ ): $164.64,153.14$, 148.46, 146.42, 143.96, 132.14, 131.32, 114.75, 102.41, 61.69, 21.36. MS: $\mathrm{M}^{+}(300)$ : Analysis (\%) for $\mathrm{C}_{15} \mathrm{H}_{16} \mathrm{~N}_{4} \mathrm{O}_{3}$ Calcd.: $\mathrm{C}$, $59.99 ; \mathrm{H}, 5.37 ; \mathrm{N}, 18.66 \%$. Found: C, 59.76; H, 5.75; N, $18.99 \%$.

3-((1-(4-Methoxyphenyl)-1H-pyrazolo[3,4-d]pyrimidin4-yl)oxy)propane-1,2-diol (4c): Yield: $91 \%$, m.p.: 175-176 ${ }^{\circ} \mathrm{C}, \mathrm{IR}\left(\mathrm{KBr}, v_{\max }, \mathrm{cm}^{-1}\right): 3440(\mathrm{OH}), 3010,2995,2878(\mathrm{CH})$, 1585, $1532(\mathrm{C}=\mathrm{C}, \mathrm{C}=\mathrm{N}),{ }^{1} \mathrm{H}$ NMR ((DMSO- $\left.d_{6}\right): \delta 3.72-3.75$ (m, $\left.3 \mathrm{H}, \mathrm{CH}_{2} \mathrm{OH}\right), 3.88\left(\mathrm{~m}, 3 \mathrm{H}, \mathrm{OCH}_{3}\right), 3.98\left(\mathrm{~s}, 3 \mathrm{H}, \mathrm{CH}_{2} \mathrm{OH}\right)$, 4.31-4.36 (m, 1H, CH), 8.39 (s, 1H, Ar-H), 8.61-8.62 (d, $J=$ $5.6 \mathrm{~Hz}, 1 \mathrm{H}, \mathrm{Ar}-\mathrm{H}), 8.77-8.78$ (d, $J=6.0 \mathrm{~Hz}, 2 \mathrm{H}, \mathrm{Ar}-\mathrm{H}), 8.87$ (s, $1 \mathrm{H}, \mathrm{Ar}-\mathrm{H}), 9.38\left(\mathrm{~s}, 1 \mathrm{H}, \mathrm{Ar}-\mathrm{H}\right.$ at $\left.\mathrm{C}_{3}\right),{ }^{13} \mathrm{C}$ NMR (DMSO- $d_{6}$ ): $163.22,152.18,148.95,146.98,143.31,133.93,131.34$, 116.64, 131.34, 116.64, 113.51, 102.01, 63.79, 61.24, 55.77. MS: $\mathrm{M}^{+}(316)$ : Analysis (\%) for $\mathrm{C}_{15} \mathrm{H}_{16} \mathrm{~N}_{4} \mathrm{O}_{4}$ Calcd.: C, 56.96; H, 5.10; N, $17.71 \%$. Found: C, 57.27; H, 5.29; N, $17.99 \%$.

3-((1-(2,4-Dinitrophenyl)-1H-pyrazolo[3,4- $d$ ]pyrimidin4-yl)oxy)propane-1,2-diol (4d): Yield: $90 \%$, m.p.: 138-139 ${ }^{\circ} \mathrm{C}, \mathrm{IR}\left(\mathrm{KBr}, v_{\max }, \mathrm{cm}^{-1}\right): 3438(\mathrm{OH}), 3010,2944,2838(\mathrm{CH})$, 1590, $1525(\mathrm{C}=\mathrm{C}, \mathrm{C}=\mathrm{N}),{ }^{1} \mathrm{H}$ NMR (DMSO- $\left.d_{6}\right): \delta 3.37-3.44$ (m, $\left.3 \mathrm{H}, \mathrm{CH}_{2} \mathrm{OH}\right), 3.79$ (s, 2H, $\left.\mathrm{CH}_{2}\right), 4.36-4.40(\mathrm{~m}, 2 \mathrm{H}, \mathrm{CHOH})$, 8.37-8.39 (d, $J=9.2 \mathrm{~Hz}, 1 \mathrm{H}, \mathrm{Ar}-\mathrm{H}), 8.78-8.79(\mathrm{~d}, J=6.0 \mathrm{~Hz}$, 2H, Ar-H), 9.29 (s, 2H, Ar-H), ${ }^{13} \mathrm{C}$ NMR (DMSO v): 164.24, 153.88, 149.07, 146.17, 143.29, 132.92, 130.98, 113.42, 102.02, 61.10. MS: $\mathrm{M}^{+}$(376): Analysis (\%) for $\mathrm{C}_{14} \mathrm{H}_{12} \mathrm{~N}_{6} \mathrm{O}_{7}$ Calcd.: $\mathrm{C}$, $44.69 ; \mathrm{H}, 3.21 ; \mathrm{N}, 22.33 \%$. Found: C, 44.77; H, 3.17; N, $22.70 \%$.

3-((1-(2,4-Dinitrophenyl)-6-methyl-1H-pyrazolo[3,4d]pyrimidin-4-yl)oxy)propane-1,2-diol (4e): Yield: $90 \%$, m.p.: $122-123^{\circ} \mathrm{C}, \mathrm{IR}\left(\mathrm{KBr}, v_{\max }, \mathrm{cm}^{-1}\right): 3444(\mathrm{OH}), 3020,2970$, $2871(\mathrm{CH}), 1585,1534(\mathrm{C}=\mathrm{C}, \mathrm{C}=\mathrm{N}),{ }^{1} \mathrm{H}$ NMR (DMSO- $\left.d_{6}\right): \delta$ 2.76 (s, $\left.3 \mathrm{H}, \mathrm{Ar}_{-} \mathrm{CH}_{3}\right), 3.76-3.89\left(\mathrm{~m}, 3 \mathrm{H}, \mathrm{CH}_{2} \mathrm{OH}\right), 3.98(\mathrm{~s}, 2 \mathrm{H}$, $\left.\mathrm{CH}_{2}\right), 4.33-4.40(\mathrm{~m}, 2 \mathrm{H}, \mathrm{CHOH}), 8.39-8.40(\mathrm{~d}, J=6.8 \mathrm{~Hz}$, $2 \mathrm{H}, \mathrm{Ar}-\mathrm{H}), 8.78-8.80(\mathrm{~d}, J=5.6 \mathrm{~Hz}, 1 \mathrm{H}, \mathrm{Ar}-\mathrm{H}), 9.16(\mathrm{~s}, 1 \mathrm{H}$, Ar-H at $\mathrm{C}_{3}$ ), ${ }^{13} \mathrm{C}$ NMR (DMSO- $d_{6}$ ): $164.85,153.85,148.16$, 146.14, 143.92, 132.65, 131.14, 114.71, 103.47, 62.42, 21.98. MS: $\mathrm{M}^{+}(390)$ : Analysis (\%) for $\mathrm{C}_{15} \mathrm{H}_{14} \mathrm{~N}_{6} \mathrm{O}_{7}$ Calcd.: C, 46.16; H, 3.62; N, $21.53 \%$. Found: C, 46.59; H, 3.23; N, $21.90 \%$.

3-((1-(Pyridin-2-yl)-1H-pyrazolo[3,4- $d$ ]pyrimidin-4yl)oxy)propane-1,2-diol (4f): Yield: $91 \%$, m.p.: $169-170{ }^{\circ} \mathrm{C}$, IR $\left(\mathrm{KBr}, v_{\max }, \mathrm{cm}^{-1}\right): 3448(\mathrm{OH}), 3020,2994,2870(\mathrm{CH}), 1587$, $1530(\mathrm{C}=\mathrm{C}, \mathrm{C}=\mathrm{N}),{ }^{1} \mathrm{H}$ NMR (DMSO- $\left.d_{6}\right): \delta 3.38-3.44(\mathrm{~m}, 3 \mathrm{H}$, $\mathrm{CH}_{2} \mathrm{OH}$ ), 3.99 (s, 2H, $\mathrm{CH}_{2}$ ), 4.33-4.40 (m, 2H, CHOH), 8.39- 
8. (d, $J=6.0 \mathrm{~Hz}, 2 \mathrm{H}, \mathrm{Ar}-\mathrm{H}), 8.78-8.80(\mathrm{~d}, J=6.4 \mathrm{~Hz}, 2 \mathrm{H}, \mathrm{Ar}-$ H), 9.28 (s, 2H, Ar-H), ${ }^{13}$ C NMR (DMSO- $d_{6}$ ): 164.78, 152.43, 149.19, 145.62, 143.24, 133.74, 130.32, 115.45, 112.14, 101.07, 63.78, 60.72. MS: $\mathrm{M}^{+}$(287): Analysis (\%) for $\mathrm{C}_{13} \mathrm{H}_{13} \mathrm{~N}_{5} \mathrm{O}_{3}$ Calcd.: C, 54.35; H, 4.56; N, $24.38 \%$. Found: C, 54.95; H, 4.46; N, $24.77 \%$.

3-((1-(Pyridin-2-yl)-6-methyl-1H-pyrazolo[3,4- $d]$ pyrimidin-4-yl)oxy)propane-1,2-diol (4g): Yield: $92 \%$, m.p.: 151-152 ${ }^{\circ} \mathrm{C}$, IR $\left(\mathrm{KBr}, v_{\max }, \mathrm{cm}^{-1}\right): 3436(\mathrm{OH}), 3015,2957$, $2838(\mathrm{CH}), 1580,1550(\mathrm{C}=\mathrm{C}, \mathrm{C}=\mathrm{N}),{ }^{1} \mathrm{H}$ NMR (DMSO- $\left.d_{6}\right): \delta$ 2.77 (s, 3H, Ar- $\left.\mathrm{CH}_{3}\right), 3.74-3.90$ (m, 3H, $\left.\mathrm{CH}_{2} \mathrm{OH}\right), 3.98$ (s, $2 \mathrm{H}$, $\mathrm{CH}_{2}$ ), 4.33-4.4 (m, 2H, CHOH), 8.39-8.40 (d, $J=6.0 \mathrm{~Hz}, 1 \mathrm{H}$, Ar-H), 8.78-8.80 (d, J = 6.4 Hz, 2H, Ar-H), 9.28 (s, 2H, Ar$\mathrm{H}),{ }^{13} \mathrm{C}$ NMR (DMSO- $d_{6}$ ): 163.11, 152.18, 148.76, 146.95, 144.71, 132.95, 131.19, 114.76, 102.19, 61.72, 21.19. MS: $\mathrm{M}^{+}$(301): Analysis (\%) for $\mathrm{C}_{14} \mathrm{H}_{15} \mathrm{~N}_{5} \mathrm{O}_{3}$ Calcd.: C, 55.81; $\mathrm{H}$, 5.02 ; N, $23.24 \%$. Found: C, 55.95; H, 5.36; N, $23.61 \%$.

3-((1-(3-Nitropyridin-4-yl)-1H-pyrazolo[3,4-d]pyrimidin-4-yl)oxy)propane-1,2-diol (4h): Yield: $88 \%$, m.p.: 152-153 ${ }^{\circ} \mathrm{C}$, IR $\left(\mathrm{KBr}, v_{\max }, \mathrm{cm}^{-1}\right): 3430(\mathrm{OH}), 3010,2978$, $2878(\mathrm{CH}), 1599,1534(\mathrm{C}=\mathrm{C}, \mathrm{C}=\mathrm{N}){ }^{1} \mathrm{H}$ NMR (DMSO- $\left.d_{6}\right): \delta$ 3.71-3.89 (m, 3H, $\left.\mathrm{CH}_{2} \mathrm{OH}\right), 3.98$ (s, 2H, $\left.\mathrm{CH}_{2}\right), 4.31-4.39$ (m, $2 \mathrm{H}, \mathrm{CHOH}), 8.38-8.39$ (d, $J=6.0 \mathrm{~Hz}, 1 \mathrm{H}, \mathrm{Ar}-\mathrm{H}), 8.77-8.79$ $(\mathrm{d}, J=6.4 \mathrm{~Hz}, 3 \mathrm{H}, \mathrm{Ar}-\mathrm{H}), 9.27$ (s, $1 \mathrm{H}, \mathrm{Ar}-\mathrm{H}$ at $\left.\mathrm{C}_{3}\right),{ }^{13} \mathrm{C} \mathrm{NMR}$ (DMSO-d $)$ : 164.17, 152.41, 149.14, 145.68, 143.73, 133.65, 130.71, 115.65, 112.14, 101.95, 63.14, 60.65. MS: $\mathrm{M}^{+}(332)$ : Analysis (\%) for $\mathrm{C}_{13} \mathrm{H}_{12} \mathrm{~N}_{6} \mathrm{O}_{5}$ Calcd.: C, 46.99; H, 3.64; N, $25.29 \%$. Found: C, 47.12; H, 3.81; N, $25.63 \%$.

3-((1-(3-Nitropyridin-4-yl)-6-methyl-1H-pyrazolo[3,4d]pyrimidin-4-yl)oxy)propane-1,2-diol (4i): Yield: $89 \%$, m.p.: $147-148^{\circ} \mathrm{C}$, IR (KBr, $\left.v_{\max }, \mathrm{cm}^{-1}\right): 3435(\mathrm{OH}), 3005,2955$, $2835(\mathrm{CH}), 1586,1550(\mathrm{C}=\mathrm{C}, \mathrm{C}=\mathrm{N}),{ }^{1} \mathrm{H}$ NMR (DMSO- $\left.d_{6}\right): \delta$ 2.6 (s, 3H, Ar- $\left.\mathrm{CH}_{3}\right), 3.71-3.88$ (m, 3H, $\left.\mathrm{CH}_{2} \mathrm{OH}\right), 3.98$ (s, 2H, $\mathrm{CH}_{2}$ ), 4.31-4.39 (m, 2H, CHOH), 8.34 (s, 1H, Ar-H), 8.878.89 (d, $J=7.2 \mathrm{~Hz}, 1 \mathrm{H}$, Ar-H), 9.1-9.12 (d, $J=8.4,2 \mathrm{H}, \mathrm{Ar}-$ $\mathrm{H}) ;{ }^{13} \mathrm{C}$ NMR (DMSO- $d_{6}$ ): 163.02, 152.63, 148.47, 146.69, 144.27, 132.14, 131.95, 114.32, 102.75, 61.98, 21.42. MS: $\mathrm{M}^{+}$(346): Analysis (\%) for $\mathrm{C}_{14} \mathrm{H}_{14} \mathrm{~N}_{6} \mathrm{O}_{5}$ Calcd.: C, 48.56; $\mathrm{H}$, 4.07; N, $24.27 \%$. Found: C, 48.78; H, 4.39; N, $24.66 \%$.

\section{RESULTS AND DISCUSSION}

(2,2-Dimethyl-1,3-dioxolan-4-yl)methanol (1) and 1,6disubstituted 4-chloropyrazolo[3,4- $d]$ pyrimidines (2) were used as starting materials for the synthesis of 1,6-disubstituted 4-(O,O-isopropylidene-2,3-dihydroxypropoxypyrazolo[3,4d]pyrimidines (3). The $\mathrm{SN}$ of chlorine in azenes normally requires a hard base like sodium hydride and a polar aprotic solvent like DMF [34] generally under heating condition. Synergism of phase transfer catalysis avoids the use of such polar and expensive solvent and use of hard base, rather it offers the use of inexpensive nonpolar solvents which are easily recovered, milder temperature condition, rapid reaction, high yield with side reaction minimization and use of low concentration of base. The SN reaction of 1,6-disubstituted 4-chloropyrazolopyrimidines (2) with (2,2-dimethyl-1,3-dioxolan-4-yl)methanol (1) was attempted under liquid-liquid phase transfer catalysis at room temperature using TBAB, chlorobenzene and aqueous $\mathrm{NaOH}$ to obtain compound $\mathbf{3}$ as off-white to yellowish white solid in 90-95\% yield. During the course of O-alkylation, there was no emulsion formation suggesting that such reactions did not follow interfacial reaction mechanism path. The lower concentration of base $(<50 \% \mathrm{w} / \mathrm{v})$ also found to lower the rate of reaction $(>24 \mathrm{~h})$. The reaction was also conducted under solid-liquid phase transfer catalysis avoiding the use of water, but liquid-liquid phase transfer catalysis was found to be more suitable with respect to yield and reaction time. Structure elucidation of all the synthesized compounds was done on the basis of IR, ${ }^{1} \mathrm{H}$ NMR, ${ }^{13} \mathrm{C}$ NMR and mass spectral analysis. In IR (KBr) spectra of 3 absorption at $3450-3400 \mathrm{~cm}^{-1}$ for $-\mathrm{OH}$ group was found to be absent indicated the complete O-heteroarylation of (2,2-dimethyl-1,3-dioxolan-4-yl)methanol. In ${ }^{1} \mathrm{H}$ NMR (DMSO- $d_{6}$ ) spectra of $\mathbf{3}$, a multiplet in the region of $\delta 1.32$ 1.34 was observed due to the presence of geminal dimethyl group in the (2,2-dimethyl-1,3-dioxolan-4-yl)methanolyl ring. A multiplet at 3.36-3.87 was responsible for $\mathrm{CH}_{2}$ protons integrating for $2 \mathrm{H}$. The protons of (2,2-dimethyl-1,3-dioxolan4-yl)methanol moiety ( $\mathrm{Ar}-\mathrm{O}-\mathrm{CH}_{2}-\mathrm{CH}-\mathrm{CH}_{2}$ ) were found to resonate at $\delta$ 4.03-4.61 in form of multiplet integrating for $5 \mathrm{H}$. Multiplet due to aromatic protons was obtained in the region $\delta 8.38-8.78$. Pyrazole proton was obtained at 9.07-9.29 giving a singlet for $1 \mathrm{H}$.

IR $(\mathrm{KBr})$ spectra of compound $\mathbf{4}$ gave absorption in the region 3448-3440 due to-OH functionality. In ${ }^{1} \mathrm{H}$ NMR (DMSO$d_{6}$ ) spectra of $4, \mathrm{CH}_{2} \mathrm{OH}$ protons for $3 \mathrm{H}$ appeared at 3.37-3.89, $\mathrm{CHOH}$ for $2 \mathrm{H}$ at 3.79-3.99 and $\mathrm{CH}_{2}$ for $2 \mathrm{H}$ at 4.31-4.40 each as multiplet. Multiplet due to aromatic protons was appeared in the region $\delta 7.24-8.19$. The data obtained is in agreement with isopropylidene ring opening of $\mathbf{3}$. Pyrazole proton was obtained at 9.07-9.38 giving a singlet for $1 \mathrm{H}$.

\section{Conclusion}

A comparison of different phase transfer catalysis conditions for C-O cross coupling in synthesis of 4-((2,2-dimethyl1,3-dioxolan-4-yl)methoxy)-1,6-disubstituted $1 H$-pyrazolo$[3,4-d]$ pyrimidines $(3)$ has been studied where liquid-liquid phase transfer catalysis using TBAB as phase transfer catalyst and a mixture of aqueous $\mathrm{NaOH}$ and chlorobenzene as a solvent at room temperature proved to be rapid and increased yield enhancing. Ring opening of $\mathbf{3}$ under acid hydrolysis at room temperature resulted the formation of 3-(1,6-disubstituted $1 \mathrm{H}$ pyrazolo[3,4-d]pyrimidin-4-yloxy)propane-1,2-diols (4) in excellent yield.

\section{ACKNOWLEDGEMENTS}

The authors are thankful to M.G. Science Institute and National Drug Discovery Center for spectral analysis.

\section{CONFLICT OF INTEREST}

The authors declare that there is no conflict of interests regarding the publication of this article.

\section{REFERENCES}

1. M. Makosza and M. Ludwikow, Angew. Chem. Int. Ed. Eng., 13, 665 (1974); https://doi.org/10.1002/anie.197406651.

2. M. Ikunaka, Org. Progr. Res. Dev., 12, 698 (2008); https://doi.org/10.1021/op700262h. 
3. S. D'Antone, M. Penco, R. Solaro and E. Chiellini, React. Polym. Ion Exchang. Sorbents, 3, 107 (1985); https://doi.org/10.1016/0167-6989(85)90054-6.

4. J. A. Zoltewicz, eds.: R.D. Rieke, J.A. Zoltewicz, H.G. Aurich, W. Weiss and K.P.C. Vollhardt, New Directions in Aromatic Nucleophilic Substitution, In: Organic Synthesis, John Wiley \& Sons Inc., SpringerVerlag: Berlin/Heidelberg, pp. 33-64 (1975).

5. K. Urata and N. Takaishi, J. Am. Oil Chem. Soc., 71, 1027 (1994); https://doi.org/10.1007/BF02542274.

6. M. Sutter, E. D. Silva, N. Duguet, Y. Raoul, E. Métay and M. Lemaire, Chem. Rev., 115, 8609 (2015) https://doi.org/10.1021/cr5004002.

7. M. Renoll and M.S. Newman, DL-Isopropylideneglycerol: Glycerol, isopropylidene-; also 1,3-dioxolane-4-methanol, 2,2-dimethyl-, In: Organic Synthesis, John Wiley \& Sons Inc., pp. 73-73 (2003). https://doi.org/10.1002/0471264180.os028.29.

8. E. De Clercq and A. Holy, J. Med. Chem., 22, 510 (1979); https://doi.org/10.1021/jm00191a010.

9. A. Behr, J. Eilting, K. Irawadi, J. Leschinski and F. Lindner, Green Chem., 10, 13 (2008); https://doi.org/10.1039/B710561D.

10. J.-F. Griffon, A. Dumas, R. Storer, J.-P. Sommadossi and G. Gosselin, Nuclos. Nucleot. Nucl. Acids, 28, 435 (2009); https://doi.org/10.1080/15257770903044531.

11. S. Bailey, M.R. Harnden, R.L. Jarvest, A. Parkin and M.R. Boyd, J. Med. Chem., 34, 57 (1991); https://doi.org/10.1021/jm00105a010.

12. C.G. Dave and R.D. Shah, J. Heterocycl. Chem., 35, 1295 (1998); https://doi.org/10.1002/ihet.5570350609.

13. C.G. Dave and R.D. Shah, Molecules, 7, 554 (2002); https://doi.org/10.3390/70700554.

14. R.D. Shah, Chem. Sustain. Dev., 19, 319 (2011).

15. N. Desai and R. Shah, Synthesis, 3275 (2006); https://doi.org/10.1055/s-2006-950206.

16. R.D. Shah and N.M. Shah, Int. Res. J. Nat. Appl. Sci., 4, 21 (2017).

17. A. Mattarei, A. Rossa, V. Bombardelli, M. Azzolini, M. La Spina, C. Paradisi, M. Zoratti and L. Biasutto, Eur.J. Med. Chem., 135, 77 (2017); https://doi.org/10.1016/j.ejmech.2017.04.034.

18. J. Hert, D. Hunziker, H. Kuehne, T. Luebbers, R.E. Martin, P. Mattei, W. Neidhart, H. Richter, M. Rudolph and E. Pinard, Phenoxymethyl Derivatives, WO/2017/037146 (2017).

19. M. Pagliaro, R. Ciriminna, H. Kimura, M. Rossi and C. Della-Pina, Angew. Chem. Int. Ed., 46, 4434 (2007); https://doi.org/10.1002/anie.200604694.
20. L.M. Toledo, N.B. Lydon and D. Elbaum, Curr. Med. Chem., 6, 775 (1999); https://doi.org/10.1002/chin.199946289.

21. L. Moity, A. Benazzouz, V. Molinier, V. Nardello-Rataj, M.K. Elmkaddem, P. de Caro, S. Thiébaud-Roux, V. Gerbaud, P. Marion and J.-M. Aubry, Green Chem., 17, 1779 (2015); https://doi.org/10.1039/C4GC02377C.

22. J.A. Kenar, Lipid Technol., 19, 249 (2007); https://doi.org/10.1002/lite.200700079.

23. V. Breu, M. Clozel, K. Burri, G. Hirth, W. Neidhart and H. Ramuz, FEBS Lett., 383, 37 (1996); https://doi.org/10.1016/0014-5793(96)00213-X.

24. A.A.-H. Abdel-Rahman, A.-A.S. El-Etrawy, A.E.-S. Abdel-Megied, I.F. Zeid and E.S.H. El Ashry, Nuclos. Nucleot. Nucl. Acids, 27, 1257 (2008); https://doi.org/10.1080/15257770802086898.

25. E.S.H. El Ashry, A.A.-H. Abdel-Rahman, N. Rashed and H.A. Rasheed, Arch. Pharm., 332, 327 (1999); https://doi.org/10.1002/(SICI)1521-4184(19999)332:9<327::AIDARDP327>3.0.CO;2-N

26. Y. Ouyang, H. Yang, P. Zhang, Y. Wang, S. Kaur, X. Zhu, Z. Wang, Y. Sun, W. Hong, Y. Ngeow and H. Wang, Molecules, 22, 1592 (2017); https://doi.org/10.3390/molecules22101592.

27. D. Bakhotmah, R. Abdul-Rahman and M. Fakhorji, World J. Org. Chem., 5, 1 (2017).

28. S. Mohana Roopan and R. Sompalle, Synth. Commun., 46, 645 (2016); https://doi.org/10.1080/00397911.2016.1165254.

29. A.E. Rashad, M. Abdelmegid, A.H. Shamroukh and F.M.E. Abdelmegeid, Org. Chemistry: An Indian J., 10, 224 (2014).

30. A.H. Abdelazeem, S.A. Abdelatef, M.T. El-Saadi, H.A. Omar, S.I. Khan, C.R. McCurdy and S.M. El-Moghazy, Eur. J. Pharm. Sci., 62, 197 (2014); https://doi.org/10.1016/j.ejps.2014.05.025.

31. F. Gatta, F. Perotti, L. Gradoni, M. Gramiccia, S. Orsini, G. Palazzo and V. Rossi, Eur. J. Med. Chem., 25, 419 (1990); https://doi.org/10.1016/0223-5234(90)90005-N.

32. C.C. Cheng and R.K. Robins, J. Org. Chem., 21, 1240 (1956); https://doi.org/10.1021/jo01117a010.

33. J.J. Baldwin, W.C. Lumma, G.F. Lundell, G.S. Ponticello, A.W. Raab, E.L. Engelhardt, R. Hirschmann, C.S. Sweet and A. Scriabine, J. Med. Chem., 22, 1284 (1979); https://doi.org/10.1021/jm00197a002.

34. C. Morrill, S. Babu, N. Almstead and Y.-C. Moon, Synthesis, 1791 (2013); https://doi.org/10.1055/s-0033-1338862. 